Steeg, L. van de, Langelaan, M., IJkema, R., Nugus, P., Wagner, C. Improving delirium care for hospitalized older patients: a qualitative study identifying barriers to guideline adherence.

\begin{tabular}{|l|l|}
$\begin{array}{l}\text { Postprint } \\
\text { Version }\end{array}$ & 1.0 \\
\hline Journal website & http://onlinelibrary.wiley.com/doi/10.1111/jep.12229/abstract \\
\hline Pubmed link & $\underline{\text { http://www.ncbi.nlm.nih.gov/pubmed/25081423 }}$ \\
\hline DOI & $10.1111 /$ jep.12229
\end{tabular}

This is a NIVEL certified Post Print, more info at http://www.nivel.eu

\title{
Improving delirium care for hospitalized older patients. A qualitative study identifying barriers to guideline adherence
}

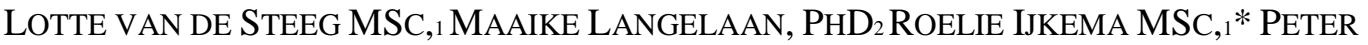 \\ Nugus PHD $2 \uparrow$ AND CORDULA WAGNER PHD ${ }_{3,4}$ \\ ${ }_{1}$ Researcher, 2 Senior Researcher, ${ }_{3}$ Program Coordinator, NIVEL, Netherlands Institute for \\ Health Services Research, Utrecht, The Netherlands \\ ${ }_{4}$ Professor, EMGO Institute for Health and Care Research, VU University Medical Centre, \\ Amsterdam, The Netherlands
}

\begin{abstract}
Rationale, aims and objectives: Guidelines are intended as a means of getting research evidence into practice and ensuring provided care is of sufficient quality. However, the effect of guidelines is hindered by limited guideline adherence. The aim of this study is to identify and classify barriers to adherence by nurses to a guideline on delirium care.

Methods: Open-ended interviews were conducted with a purposive sample of 63 research participants. The sample included 28 nurses, 18 doctors and 17 policy advisors working in 19 hospitals in the Netherlands. The interviews were conducted between June and September 2011. The data were analysed using thematic analysis.

Results: Barriers to guideline adherence that were identified could be grouped into four themes: motivation and goals, knowledge and skills, professional role and identity, and context and resources. While the interviews with nurses, doctors and policy advisors produced similar views of the current situation, doctors and policy advisors placed a higher importance on education as a means of stimulating adherence.

Conclusions: This study illustrates that individual, social and organizational factors play a role in nurse's adherence to a delirium guideline. The potential benefits of following a guideline, both for patients and for nursing staff, need to be highlighted in order to motivate nurses. When formulating new guidelines, nurses' perceptions of their professional role and patient care need to be taken into account to ensure that policy makers and managers are realistic about guideline adherence and engage with nurses from a position of mutual respect and trust.
\end{abstract}


Steeg, L. van de, Langelaan, M., IJkema, R., Nugus, P., Wagner, C. Improving delirium care for hospitalized older patients: a qualitative study identifying barriers to guideline adherence.

Journal of Evaluation in Clinical Practice: 2014, 20(6), 813-819

\section{INTRODUCTION}

Studies have shown the difficulty of persuading health care professionals to adhere to guidelines regarding the provision of care [1-3]. These guidelines are put forward as a means of getting research evidence into practice and are generally aimed at ensuring that the provision of care is of sufficient quality [4,5]. Why is it that health care professionals show reluctance in following guideline recommendations? Most studies have focused on barriers to doctor's adherence to guidelines rather than to nurses and other health care professionals [6]. Yet considerable consequences can be expected to flow from nurses' adherence or failure to adhere to guidelines. Nurses attend most frequently to patients, given their role and location in immediate care duties by the bedside. Despite increased knowledge about factors influencing guideline adherence and implementation, few clear answers have been given to the question of how to successfully introduce clinical guidelines into practice [3,7]. The opportunity for the current examination of guideline adherence was provided by a national patient safety programme in the Netherlands, which resulted in a guideline on care for older hospitalized patients being delivered in 2009 [8]. This was aimed at care for patients aged 70 and over, and included recommendations regarding delirium care. The guideline was based upon existing evidence regarding care for older patients, as well as expert opinion. The guideline recommends that delirium care includes nursing activities, such as screening patients for the risk of developing delirium, assessing patients for delirium, initiating preventative measures and supporting treatment (Box 1). The Dutch Health Care Inspectorate found that in 2010 only $56 \%$ of hospital wards screened $80 \%$ or more of the older patients for the risk of developing delirium [9]. Of the patients at risk, only 50\% were monitored for the presence of delirium using an assessment instrument, such as the Delirium Observation Screening Scale (DOSS) [10]. This indicates that, although an evidenceinformed guideline was available for delirium care, nurses do not follow it consistently. Similar results have been found for health care professionals in the United States [11,12].

\section{[воX 1]}

Research indicates that the focus on barriers to guideline adherence ought to be more broadly based, showing that barriers can be found at several levels [6,13-15]. A combined focus on older people and nurses provides an opportunity to test this broadly based focus on guideline adherence. Older people comprise a large proportion of the patient population worldwide, and this proportion is only expected to increase [16]. This implies that looking specifically at guidelines aimed at older patients and the possible barriers that play a role in adherence to these guidelines is of importance for quality of health care now and will become even more so in the future. Furthermore, older people are more likely than younger people to suffer multiple conditions simultaneously, creating greater risks from treatment that is poorly coordinated [17], as well as from possible conflicting guidelines.

This paper focuses, in particular, on nurses' behaviour regarding delirium care for older patients and the factors that influence that behaviour, as a window onto their adherence to guidelines. If guidelines are valued by policy makers, we need to know the range of barriers to guideline adherence and be able to conceptualize them. The aim of this study was to identify and classify barriers to guideline adherence by 
Steeg, L. van de, Langelaan, M., IJkema, R., Nugus, P., Wagner, C. Improving delirium care for hospitalized older patients: a qualitative study identifying barriers to guideline adherence.

Journal of Evaluation in Clinical Practice: 2014, 20(6), 813-819

nurses, by exploring the perceptions of nurses and other professionals regarding the implementation of a guideline on delirium care.

\section{METHODS}

\section{Study design and setting}

This qualitative study was conducted between June and September 2011 and consisted of 63 open-ended interviews. We chose this approach because interviews can offer insight into experiences and attitudes, as well as intentions that inform people's behaviours [18]. This qualitative study was part of a larger research project focusing on the effect of e-learning on delirium care for older patients: a stepped wedge trial on the effect of e-learning on delirium care [19].

Data were gathered in 19 Dutch hospitals, which included 2 university hospitals, 6 tertiary teaching hospitals and 11 general hospitals, varying in size (from 138 to 1230 beds) and geographical location. The hospitals that responded to the invitation within the inclusion period were included in the study, provided they had already started working according to the government-sanctioned guideline of care for older patients [8]. This criterion was chosen to ensure that the hospitals would be able to supply relevant data. Nearly all hospitals in the Netherlands were initially invited (81) to participate in the study, excluding specialized hospitals such as eye clinics and cancer hospitals, and 10 hospitals already participating in a different study concerning care for older patients. Thirty hospitals responded positively to the invitation, six of which responded outside the inclusion period. Five hospitals declined to participate after receiving more information about the study - one after already being enrolled in the study - mostly due to time constraints.

\section{Participants}

Interview participants were selected by asking the contact person for the study in each hospital, generally a policy advisor or team leader, to provide the research team with the names of persons willing to participate. The aim was to interview at least one doctor, one nurse and one policy advisor per hospital, all with experience regarding care for older patients, either directly or indirectly. Policy advisors were employed by the hospital and involved with developing, implementing and monitoring hospital policy. While some had a clinical background, this was not necessarily the case.

Our goal was to speak with individuals who could provide information and perspectives on processes and practices in the hospital for delivering care to older patients.

Some contact persons suggested that in order to gain a more rounded view of care for older patients in their hospital, an interview with a fourth person was necessary. If this was the case, such an additional participant was also approached. We chose a wide selection of professionals, from a large number of hospitals, in order to gain insight into the different experiences with, and diversity of opinions on, care for older patients and the guideline on delirium care. Clinicians were recruited both from general medical and surgical wards and from specialty geriatric units. On average, 3.5 persons per hospital were interviewed, with three being the minimum and five the maximum number of participants for a single hospital. See Table 1 for the sample of interviewed health care professionals. 
Steeg, L. van de, Langelaan, M., IJkema, R., Nugus, P., Wagner, C. Improving delirium care for hospitalized older patients: a qualitative study identifying barriers to guideline adherence.

Journal of Evaluation in Clinical Practice: 2014, 20(6), 813-819

\section{[TABLE 1]}

\section{Data collection}

The open-ended, semi-structured interviews were conducted by two researchers (RI and LS) using a topic guide based upon a review of implementation literature [15]. For policy advisors, the focus was on their experiences with introducing the guideline on care for older patients into their hospital. Nurses, policy advisors and doctors were questioned to gauge their perspectives on the behaviour and attitude of nurses regarding care for older patients and the delirium guideline, as well as their own experiences.

All participants were initially approached by the hospital's contact person for the study, with whom the researchers maintained contact during the research period. The contact person and subsequently the researcher conducting the interview explained the study aims and methods. The participants were assured that their individual comments would remain confidential. Research ethics committee approval was obtained for the study protocol of the larger research project, which included this qualitative study.

Interviews were conducted until data saturation was reached, meaning that no new ideas or themes emerged during the interviews [20]. Overall, the interviews lasted between 30 and 75 minutes. They were audio-taped and later transcribed by the researchers. Three participants were interviewed by phone, for which transcription was undertaken by hand. Reliability of transcription was enhanced by returning the transcripts to the participants for verification and comment [21]. The interviews took place at a location convenient for the participant, generally a meeting room or office in their hospital. The interviews were conducted and analysed in Dutch.

\section{Data analysis and rigour}

The interview transcripts were coded by the two researchers who conducted the interviews, using MAXQDA 2007 (VERBI). A coding structure was developed by these researchers prior to coding, to facilitate the thematic qualitative analysis. This structure was based upon the theoretical background that was used when assembling the topic guide and other implementation literature [15,22].

The coding structure was expanded and modified after both researchers independently coded the same interview transcript and discussed the differences in assignment of codes and interpretation of codes [23]. Following the coding of all transcripts, the researchers each independently coded five transcripts that had already been coded by the other researcher. For these 10 transcripts, the double set of codes were discussed until consensus was reached [24]. This led to a further modification of the coding structure. Subsequently, all coded transcripts were reviewed and checked against the final coding structure. Codes were then categorized and themes were identified.

\section{RESULTS}

In the following section, the findings from the interviews will be presented, grouped into four themes that emerged from the findings. These themes provided insight into the behaviour of nurses concerning the Dutch guideline on delirium care: motivation and goals, knowledge and skills, professional role and identity, and context and resources. 
Steeg, L. van de, Langelaan, M., IJkema, R., Nugus, P., Wagner, C. Improving delirium care for hospitalized older patients: a qualitative study identifying barriers to guideline adherence.

Journal of Evaluation in Clinical Practice: 2014, 20(6), 813-819

\section{Motivation and goals}

The interviews indicated that overall, nurses lack motivation to use the risk screening instrument, despite the recommendation in the guideline. This is mainly influenced by the apparent lack of clarity of the benefits and goals of screening. As a result, nurses view screening as simply completion of another registration form, encroaching on the time available for patient care.

My experience is that nurses are quick to say: 'Oh, not more [registration]' because it always comes at the expense of patient care. And of course it is a lot they have to do, filling in all these forms. (Geriatric nurse hospital 9)

Nursing team leaders in particular suggested that convincing nurses of the benefits of screening would be easier if evidence of these benefits was available. Studies showing the effect of the risk screening on patient outcomes are believed not to be widely available. The results of screening are not directly visible for nurses either. Most hospitals do not have data available regarding their performance on delirium care, making it impossible to show whether the screening of patients leads to better overall patient outcomes.

The readiness to change is there as soon as the nurses see the benefits themselves. A delirious patient takes up a lot of time. (...) You will notice few results of the implementation in the short term, only long term will more results become evident. This complicates the implementation. (Geriatric nurse hospital 12)

Doctors had a similar view, recognizing that nurses are often initially sceptical towards risk screening, mainly because of the expectation that screening will increase their workload. Like nursing team leaders, doctors felt that nurses needed to be properly informed of the benefits of risk screening in order to motivate them. More importantly, nurses need to experience these benefits first-hand.

Besides the lack of time, I think that a lack of awareness and knowledge of health problems in older persons and the importance of screening contributes to the screening not taking place as planned. Nurses rarely see direct effects of the screening taking place or not, making it difficult to connect screening to the end goal. (Geriatrician hospital 8)

Besides the advantages of screening being unclear, nurses did not always experience a need for changing delirium care and introducing screening: they felt that care for delirious patients was already optimal. The risk screening as a whole was seen as an obligation, imposed on the nursing staff from the 'top down'. Nurses would screen patients not because they thought it useful but because they had to. DOSS, for instance, is a lot of work to fill in correctly and not every nurse sees the value of that instrument. They'll say: 'I can see for myself whether a patient is confused'. While at the same time after a fall incident it will become clear that the patient had a very high DOSS score, which had not resulted in any actions. (Nurse team leader internal medicine hospital 1)

\section{Knowledge and skills}

The level of knowledge nurses reported concerning the guideline and the larger patient safety programme that it stems from differed greatly. Team leaders and nurses specialized in care for older patients showed greater knowledge about the guideline and its background than most nurses from other medical and surgical units. Risk screening was the aspect of the guideline that most nurses were familiar with. In general, nurses conveyed that they had sufficient knowledge and skills to use the screening instrument to identify at risk patients, or the DOSS in order to observe 
Steeg, L. van de, Langelaan, M., IJkema, R., Nugus, P., Wagner, C. Improving delirium care for hospitalized older patients: a qualitative study identifying barriers to guideline adherence. Journal of Evaluation in Clinical Practice: 2014, 20(6), 813-819

possibly delirious patients. This perception is supported by the fact that these instruments were developed for use by nurses without specific training. Whether nurses considered themselves capable of taking subsequent preventive or treatment measures is less clear: some indicated high levels of confidence, whereas others had doubts about their own and others' level of knowledge.

I believe I have sufficient knowledge regarding delirium: theoretical knowledge from my formal education and knowledge from personal experience (...). My colleagues are well informed about delirium. They are quick to recognize a delirious patient based on their behaviour and the (irregular) answers they give when questioned. (Orthopaedic nurse hospital 6)

During the interviews, it became clear that delirious patients put pressure on nursing staff. They require extra attention from nurses and can also make nurses feel as if they are practicing unsafely. Participants believed that nurses who had experienced the difficulty of coping with these patients on the ward were open to advice on how to handle delirious patients.

Nurses are open to training on delirium care, because care for delirious patients can be very difficult and nurses appreciate learning how to deal with these patients better. They [delirious patients] are very difficult [patients], so if you experience a lot of impotence it is no good just being told to act professionally. You have to help people [nurses] of course. (Geriatric nurse hospital 15)

Doctors mainly emphasized the importance of additional education for nurses on delirium screening and treatment.

Working with the DOSS is sometimes difficult for nurses. Extra education on this subject is needed. Nurses are inclined to directly judge from their observations, while the DOSS requires the observations to be scored without judgment or interpretation. (psychiatrist hospital 8)

One doctor suggested that a lack of knowledge about delirium care might increase the use of pharmacological interventions or patient restraints because nurses are insufficiently aware of the non-pharmacological interventions at their disposal.

\section{Context and resources}

The social pressure to screen all older patients appears to be limited: it is generally accepted among nurses that other activities take precedent over screening. This finding is confirmed by the search of nurse team leaders for ways in which screening can be enforced.

It is clear that it still is difficult for nurses to complete the whole medical history, including risk screening, for every older patient. This is probably part of the culture. On the wards we do several things to try to change this, such as starting up a competition. Every week we check which percentage of older patients had a complete medical history. The team with the lowest percentage has to buy the others cake. The competition element helps increase the completion of the medical history of patients. (Nurse team leader internal medicine hospital 1)

An important factor influencing nurses' behaviour regarding the guideline was the perceived time nurses have to invest in screening and other activities, such as observing patients using the DOSS. Nurses report having a shortage of time in which to perform their day-to-day tasks, leading to the professed inability to perform timeconsuming or perceived non-essential activities such as screening or observing patients using the DOSS. This perception of nurses was not conducive to a positive attitude towards changing delirium care. 
Steeg, L. van de, Langelaan, M., IJkema, R., Nugus, P., Wagner, C. Improving delirium care for hospitalized older patients: a qualitative study identifying barriers to guideline adherence.

Journal of Evaluation in Clinical Practice: 2014, 20(6), 813-819

Concerning the screening, nurses are quick to say: 'We are already very busy and we already have to fill in so many lists. However, this is nothing new: nurses have complained about lack of time before this project and they will probably be complaining in the future. (...) It really is difficult; there are so many measurements that need to be taken for one patient. And you don't have one patient, you have 38 on a ward. It just is a lot. (Nurse team leader surgery hospital 8)

The fact that policy advisors generally work for the entire hospital rather than a specific ward or specialty might help them develop a broader view of delirium care. Policy advisors indicated during the interviews that a striking feature of delirium care, for them, was the difference between wards. While some wards were highly receptive to the introduction of risk screening, other wards were less open to this change. The presence of enthusiastic leaders and the experience with delirium care on the ward were factors that the policy advisors identified as influential.

The ease with which a protocol is translated into a different way of working differs strongly per ward. On wards such as neurology, many of whose patients are delirious, a new protocol is easily picked up by nurses because they recognize its importance. (Policy advisor hospital 10)

If a team leader 'gets' the subject, the subject gets attention on the ward. (...) I see it here, with a team leader who was part of the project team; she is also very actively involved with the subject on her own ward. (...) The team leader is the pivot on which everything hinges: the wards that are furthest along have a team leader who makes an effort. (Policy advisor hospital 1)

As well as the doctors, the policy advisors were convinced of the importance of education for nursing staff, especially to increase awareness. However, they indicated that to change nurses' behaviour, education alone was not sufficient. They believed that the effects of formal education wear off over time or are negated by the effects of their working environment, after which old habits reappear.

Nurses understand the choice for the new way of working, but when on their wards they do fall back on old methods and patterns. Within the hospital we are still looking for a way to really make the new way of working sink in with the nurses and make them enthusiastic about it. (Policy advisor hospital 1)

The presence of other projects in the hospitals or other changes taking place play a role in the attitude and behaviour of nurses regarding delirium care and changes made to this type of care. Policy advisors suggested that the introduction of an electronic patient record (EPR) influenced the introduction of risk screening, both positively and negatively. Using the introduction of the EPR as a way of including screening instruments in the regular medical examination also meant using it as a way of stimulating screening. An additional advantage of working with an EPR, expressed by policy advisors, is the data on performances and patient outcomes that can be generated. They expect this will make it possible to show nurses the benefits of changing the care they provide, with the intention of positively influencing the motivation of nurses regarding screening in particular.

The hospital is working on an EPR. I hope that this system will lighten the load for nurses, for instance by showing certain measurements and follow-up actions automatically so nurses don't have to keep track of these actions themselves. By making certain fields for registration compulsory in the electronic record, nurses can be supported as well. Because of time pressure, measurements or registrations are 
Steeg, L. van de, Langelaan, M., IJkema, R., Nugus, P., Wagner, C. Improving delirium care for hospitalized older patients: a qualitative study identifying barriers to guideline adherence.

Journal of Evaluation in Clinical Practice: 2014, 20(6), 813-819

sometimes skipped, with the intention of doing them later, which is often subsequently forgotten. (Policy advisor hospital 4)

On the other hand, some argued that the introduction of the EPR required so much attention and time of the nursing staff that delirium care and screening suffered for it. This is supported by the view expressed by some participants that nurses have been and are being confronted with such a great number of changes that they are unable to keep up and fail to fully incorporate these changes in their routine practice.

\section{Professional role and identity}

Nurses view screening as not being part of the essential care for older persons. This is exemplified by the fact that nurses report that screening is one of the first activities they omit when time is limited.

The project regarding care for older patients was well received; it filled a need. But [computer work] is also the first thing you drop: patient care always comes before computer work. Your report needs to be finished for the next shift and the things you abandon first are scores, check lists or registration lists. (Internal medicine nurse hospital 18)

Not only did these nurses not consider delirium risk screening to be an intrinsic part of their job, some nurses also disagreed with the content of the guideline. The screening instrument is seen as limiting their autonomy, while they have little faith in the usefulness of the instrument itself.

I prefer using my clinical observation instead of a score. I'm sure the three questions on delirium are theoretically sound, but in practice they are useless. The question regarding self-care for instance: there are so many people that need help with selfcare activities, but even if that is the only question answered positively, you have to use the DOSS three times a day! That is not motivating. (Internal medicine nurse hospital 16)

Nurses believe that the screening instrument and DOSS strengthen their position when communicating with doctors. It provides them with a way to clearly indicate the risks or symptoms identified. The increased credibility it gives nurses is believed to stimulate use of the guideline.

A negative influence on delirium care is the general view from participants that most nurses on general wards consider care for older patients to be uninteresting or 'not exciting'. Several doctors concurred with this notion. This type of care is viewed by nurses as 'basic care', as opposed to more attractive highly specialized care such as oncology or intensive care nursing.

There are nurses that are interested in older patients or care for older patients and then there are people who simply say out loud, how horrible they find it. (Geriatric nurse consultant hospital 15)

There are surgical wards where the nursing staff believe these types of patients do not belong on their ward. The majority of the staff do not think care for older persons is interesting, and believe these patients do not belong in hospital. (Internist hospital 12)

Nurses like having been given more resources. Added to this they don't have to argue with doctors anymore. If a patient meets a certain criterion, then medication has to be started. The protocol has become more clear, which leads to less argument. (Internal medicine nurse hospital 13) 
Steeg, L. van de, Langelaan, M., IJkema, R., Nugus, P., Wagner, C. Improving delirium care for hospitalized older patients: a qualitative study identifying barriers to guideline adherence.

Journal of Evaluation in Clinical Practice: 2014, 20(6), 813-819

\section{DISCUSSION}

This qualitative interview study explored the gap between formal guidelines and their adherence in practice, drawing on the case of delirium care in Dutch hospitals. In addition to previous knowledge of barriers to guideline adherence, this study illustrates that individual, social and organizational factors play a role in nurse's adherence to a delirium guideline. Barriers were identified regarding motivation and goals, knowledge and skills, professional role and identity, and context and resources. The findings show that some barriers were relatively clear-cut, such as a lack of understanding of the potential benefits of working according to the guideline, where others were more complex, touching upon differing views of the professional roles of nurses. Predominantly, the study showed the interplay between behaviour and context that habituates behaviour, the latter of which needs to be taken into account if guideline adherence is to be a realistic prospect in day-to-day work. Our findings show that nurses often equated screening with registration, not recognizing the actual goal of identifying at-risk patients and being able to provide preventative care. The supposed lack of evidence supporting the screening instrument in some quarters is perceived to exacerbate the weak motivation of nurses regarding screening. Given nurses' relative autonomy and professional accountability, the fact that they would question the need to follow this guideline might not come as a surprise. That this critical attitude of nurses is viewed as a problem by the hospital or their colleagues confirms the findings of Bail et al. [25], who found that hospitals expect their nursing staff to be obedient to hospital policy and requirements, while the nursing profession expects nurses to be autonomous professionals with their own clinical judgment.

Furthermore, nurses feel that the guideline has to be followed as is, not leaving room for specific situations in which the patients' needs justify deviating from the guideline. This perceived inflexibility of a guideline in dealing with the complex reality of clinical care has been recognized in other studies [25-27]. While policy advisors expect the introduction of an EPR to improve the adherence to the guideline, this could also increase nurses' dissatisfaction with the guideline by further limiting the autonomy of nurses.

In the United States, the use of a reliable and valid instrument for delirium assessment in intensive care units was hindered by a lack of time and confidence in using the instrument [12]. We also found that time played an important role in the Dutch context: nurses who already experienced a shortage of time felt that applying the guideline required them to invest even more time in each patient. Even more important seems to be the view of nurses of risk screening, and to a lesser extent using the DOSS, as being non-essential parts of patient care. Nurses view these as aspects of care that can be pushed aside when time is limited. In addition, we found that there is a sense among nursing staff that caring for older patients is not their core business. Similarly, Teodorczuk and colleagues found a lack of ownership of confused older patients in hospital staff, as well as negative attitudes towards this patient group [28]. How health care professionals define their work and which core activities make up their profession influences how those professionals respond to certain guidelines and patient groups.

Most nurses believed that they and their colleagues possessed sufficient knowledge at least to identify at-risk and delirious patients using these instruments. Working according to the guideline even increased nurses' confidence when communicating 
Steeg, L. van de, Langelaan, M., IJkema, R., Nugus, P., Wagner, C. Improving delirium care for hospitalized older patients: a qualitative study identifying barriers to guideline adherence. Journal of Evaluation in Clinical Practice: 2014, 20(6), 813-819

with doctors. Like Manias and Street [26], we found that nurses used parts of the guideline - the screening instrument and DOSS - to assert power and legitimize their actions. This fits in with findings from other studies, indicating either that nurses feel that doctors pay more attention to structured delirium assessments, or that doctors lack respect for nurses' clinical observations [29,30].

Delirious patients are often viewed by nursing staff as potential risks to the safety of other patients as well as staff, causing the care for these patients to be challenging and often stressful [31-33]. That nurses in this study differed in how confident they were in caring for delirious patients and often welcomed advice, fits in with this view. Doctors and policy advisors identified education of nurses on delirium as an important way of increasing adherence to the guideline in this study. Education on delirium care could increase nurses' confidence when caring for delirious patients, as well as inform nurses about the goals and benefits of working according to the guideline.

\section{Limitations}

This study has some limitations. The interview participants were selected by the hospital contact person, which lead to the researcher not being aware of any potential participants refusing. It is possible that a selection bias was introduced by using this way of selecting and approaching participants. As a qualitative study undertaken in one country only, the findings might not be generalizable to other settings. However, despite differences between countries regarding health systems, we found many similarities between the experiences and attitudes of Dutch nursing staff and of nurses in the UK, the United States and Australia [12,25-30]. This seems to suggest that our findings can be transferred to other countries, at least in the Western world. Overall, the study showed the case of nursing care of delirium patients to be a case study of how health care professionals' intentions towards guidelines are formed. This information can be useful for further study into the factors influencing guideline adherence.

\section{CONCLUSION}

This study shows how varied and sometimes complex the barriers can be, which hinders guideline adherence. Barriers were found concerning the motivations of nurses, their knowledge and skills, nurses' perception of their professional roles and identities, and finally the context into which the guideline was introduced, as well as the resources available to nursing staff.

When introducing a new way of working, either through a guideline or different method, the potential benefits of changing professional behaviour need to be highlighted, for example, through education and immediate feedback of results. Policy makers need to take nurses' perceptions of their professional roles and of patient care into account when formulating new guidelines to ensure expectations of guideline adherence are realistic and nurses' autonomy is respected. Otherwise, resistance to the guideline is to be expected, as well as a lack of motivation. Collectively, redressing the barriers to guidelines adherence involves a partnership between the contexts of policy, practice cultures and education to maximize the quality of care for older patients.

\section{Acknowledgements}

The authors would like to thank all the interviewees for their participation. 
Steeg, L. van de, Langelaan, M., IJkema, R., Nugus, P., Wagner, C. Improving delirium care for hospitalized older patients: a qualitative study identifying barriers to guideline adherence.

Journal of Evaluation in Clinical Practice: 2014, 20(6), 813-819

\section{REFERENCES}

1Cabana, M. D., Rand, C. S., Powe, N. R., Wu, A. W., Wilson, M. H., Abboud, P. A. \& Rubin, H. R. (1999) Why don't physicians follow clinical practice guidelines? A framework for improvement. Journal of the American Medical Association, 282 (15), 1458-1465.

2Davison, T. E., Snowdon, J., Castle, N., McCabe, M. P., Mellor, D., Karantzas, G. \& Allan, J. (2012) An evaluation of a national program to implement the Cornell Scale for Depression in Dementia into routine practice in aged care facilities. International Psychogeriatrics, 24 (4), 631-641.

3Koh, S. S. L., Manias, E., Hutchinson, A. M., Donath, S. \& Johnston, L. (2008) Nurses' perceived barriers to the implementation of a fall prevention clinical practice guideline in Singapore hospitals. BMC Health Services Research, 8, 105.

4Buchan, H., Sewell, J. R. \& Sweet, M. (2004) Translating evidence into practice. The Medical Journal of Australia, 180, S43-S44.

5Grol, R. \& Grimshaw, J. (2003) From best evidence to best practice: effective implementation of change in patients' care. Lancet, 362, 1225-1230.

6 Abrahamson, K. A., Fox, R. L. \& Doebbeling, B. N. (2012) Facilitators and barriers to clinical practice guideline use among nurses. The American Journal of Nursing, 112 (7), 26-35.

7Francke, A. L., Smit, M. C., de Veer, A. J. E. \& Mistiaen, P. (2008) Factors influencing the implementation of clinical guidelines for health care professionals: a systematic metareview. BMC Medical Informatics and Decision Making, 8, 38.

8VMS (National Patient Safety Program) (2009) Frail Elderly [in Dutch]. Available at http://www.vmszorg.nl/_page/vms_inline?nodeid=7001\&subjectid=6702 (last accessed 8 July 2014).

9IGZ (Dutch Healthcare Inspectorate) (2012) The Results Count Hospitals 2010. Utrecht: IGZ.

10Van Gemert, L. A. \& Schuurmans, M. J. (2007) The Neecham Confusion Scale and the Delirium Observation Screening Scale: capacity to discriminate and ease of use in clinical practice. BMC Nursing, 6, 3.

11Ely, E. W., Stephens, R. K., Jackson, J. C., Thomason, J. W., Truman, B., Gordon, S., Dittus, R. S. \& Bernard, G. R. (2004) Current opinions regarding the importance, diagnosis, and management of delirium in the intensive care unit: a survey of 912 healthcare professionals. Critical Care Medicine, 32 (1), 106-112.

12Pun, B. T., Gordon, S. M., Peterson, J. F., Shintani, A. K., Jackson, J. C., Foss, J., Harding, S. D., Bernard, G. R., Dittus, R. S. \& Ely, E. W. (2005) Large-scale implementation of sedation and delirium monitoring in the intensive care unit: a report from two medical centers. Critical Care Medicine, 33 (6), 1199-1205.

13Bosch, M., Van der Weijden, T., Wensing, M. \& Grol, G. (2007) Tailoring quality improvement interventions to identified barriers: a multiple case analysis. Journal of Evaluation in Clinical Practice, 13, 161-168.

14Francis, J. J., O'Connor, D. \& Curran, J. (2012) Theories of behaviour change synthesised into a set of theoretical groupings: introducing a thematic series on the theoretical domains framework. Implementation Science, 24, 7-35.

15Grol, R. \& Wensing, M. (2011) Implementatie, effectieve verandering in de patiëntenzorg. Amsterdam: Reed Business.

16Christensen, K., Doblhammer, G., Rau, R. \& Vaupel, J. W. (2009) Ageing populations: the challenges ahead. Lancet, 374 (9696), 1196-1208.

17Leichsenring, K. (2004) Developing integrated health and social care services for older persons in Europe. International Journal of Integrated Care, 4, e10.

18Pope, C., van Royen, P. \& Baker, R. (2002) Qualitative methods in research in healthcare quality. Quality and Safety in Health Care, 11, 148-152.

19 van de Steeg, L., ljkema, R., Langelaan, M. \& Wagner, C. (2014) Can an e-learning course improve nursing care for older people at risk of delirium: a stepped wedge cluster randomised trial. BMC Geriatrics, 14, 69.

20Charmaz, K. (2000) Grounded theory: objectivist and constructivist methods. In Handbook of Qualitative Research (eds N. K. Denzin \& Y. S. Lincoln), pp. 509-536. Thousand Oaks, CA: Sage. 
Steeg, L. van de, Langelaan, M., IJkema, R., Nugus, P., Wagner, C. Improving delirium care for hospitalized older patients: a qualitative study identifying barriers to guideline adherence.

Journal of Evaluation in Clinical Practice: 2014, 20(6), 813-819

21Creswell, J. W. \& Miller, D. L. (2000) Determining validity in qualitative inquiry. Theory into Practice, 39 (3), 124-130.

22Pettigrew, A. M. \& Whipp, R. (1993) Managing Change for Competitive Success. Oxford: Blackwell Publishers.

23Silverman, D. (2010) Doing Qualitative Research, 3rd edn. London: Sage.

24Driedger, S. M., Gallois, C., Sanders, C. B. \& Santesso, N. (2006) Finding common ground in team-based qualitative research using the convergent interviewing method. Qualitative Health Research, 16 (8), 1145-1157.

25Bail, K., Cook, R., Gadner, A. \& Grealish, L. (2009) Writing ourselves into a web of obedience: a nursing policy analysis. International Journal of Nursing Studies, 46, 14571466.

26Manias, E. \& Street, A. (2000) Legitimation of nurses' knowledge through policies and protocols in clinical practice. Journal of Advanced Nursing, 32 (6), 1467-1475.

Wiley Online Library |

27Mead, P. (2000) Clinical guidelines: promoting clinical effectiveness or a professional minefield? Journal of Advanced Nursing, 31 (1), 110-116.

Wiley Online Library |

28Teodorczuk, A., Mukaetova-Ladinska, E., Corbett, S. \& Welfare, M. (2013)

Reconceptualizing models of delirium education: findings of a Grounded Theory study. International Psychogeriatrics, 25 (4), 645-655.

29Eastwood, G. M., Peck, L., Bellomo, R., Baldwin, I. \& Reade, M. C. (2012) A questionnaire survey of critical care nurses' attitudes to delirium assessment before and after introduction of the CAM-ICU. Australian Critical Care, 25 (3), 162-169.

30 Hosie, A., Lobb, E., Agar, M., Davidson, P. M. \& Philips, J. (2014) Identifying the barriers and enablers to palliative care nurses' recognition and assessment of delirium symptoms: a qualitative study. Journal of Pain and Symptom Management. doi: 10.1016/j.jpainsymman.2014.01.008.

31Schofield, I., Tolson, D. \& Fleming, V. (2012) How nurses understand and care for older people with delirium in the acute hospital: a critical discourse analysis. Nursing Inquiry, 19 (2), 165-176.

32Poole, J. \& Mott, S. (2003) Agitated older patients: nurses' perceptions and reality. International Journal of Nursing Practice, 9, 306-312.

33Agar, M., Draper, B., Phillips, P. A., Phillips, J., Collier, A., Harlum, J. \& Currow, D. (2012) Making decisions about delirium: a qualitative comparison of decision making between nurses working in palliative care, aged care, aged care psychiatry, and oncology. Palliative Medicine, 26 (7), 887-896. 
Steeg, L. van de, Langelaan, M., IJkema, R., Nugus, P., Wagner, C. Improving delirium care for hospitalized older patients: a qualitative study identifying barriers to guideline adherence. Journal of Evaluation in Clinical Practice: 2014, 20(6), 813-819

\section{TABLES AND BOXES}

Box 1 Summary of Dutch guideline on delirium care (VMS, 2009) Risk screening for all patients aged 70 and over.

Three questions for the patient and/or family or caregivers, asked by nursing staff:

- Do you experience memory problems?

- Have you needed help with self care in the last 24 hours?

- Have you experienced periods of confusion during earlier hospital stay or illness?

One or more questions answered with 'yes' indicates a risk of developing delirium.

Possible nursing interventions

- Observation with the Delirium Observation Screening Scale

- Prevent dehydration, infections, electrolyte disturbances, etc.

- Adequate treatment of pain

- Preserve nutritional level

- Inform patients' family

- Improve sensory perception

- Restrict restraining patients mechanically

Possible medical interventions

- Review medication

- Prevent dehydration, infections, electrolyte disturbances, etc.

- Adequate treatment of pain

- Consider stopping the use of invasive interventions

- Restrict restraining patients mechanically

- Provide a circadian rhythm

- Consult geriatrics

Recommendations regarding implementation

- Create a sense of urgency

- Gather a project team together

- Develop a plan of action, containing vision and strategy

- Communicate that vision and strategy to others

- Remove obstacles to following the guideline

- Create short term, visible, successes

- Continue pursuing change after the first results have been had

- Make the new situation part of the organizational culture

- Incorporate the risk screening in medical history forms

- Incorporate the recommendations from the guideline in the hospital delirium protocol 
Steeg, L. van de, Langelaan, M., IJkema, R., Nugus, P., Wagner, C. Improving delirium care for hospitalized older patients: a qualitative study identifying barriers to guideline adherence. Journal of Evaluation in Clinical Practice: 2014, 20(6), 813-819

\section{[TABLE 1]}

Health care professionals interviewed about delirium care for older patients

\begin{tabular}{||l|l|}
\hline \multicolumn{1}{|c|}{ Health care professionals } & Number \\
\hline Doctors (surgeon, internist, psychiatrist) & 7 \\
\hline \hline Geriatricians & 11 \\
\hline Nurses (from surgical and internal medicine wards) & 14 \\
\hline Nurse team leaders & 4 \\
\hline \hline Geriatric nurses & 10 \\
\hline \hline Policy advisors & 17 \\
\hline Total & 63 \\
\hline
\end{tabular}

